\title{
Grußworte zur Eröffnung des Medientags 2019
}

\author{
Hermann Petz, Vorstandsvorsitzender der Moser Holding AG
}

Sehr geehrter Herr Rektor, sehr geehrte Herren Professoren Hug und Pallaver, liebe VeranstaltungsteilnehmerInnen,

der Medientag 2019 stand unter dem Motto der Jugendlichen Mediennutzung als Herausforderung für den Qualitätsjournalismus. Allein der Titel lädt zu angeregten Diskussionen ein. Denn sowohl der Qualitätsbegriff von Journalismus - im Rahmen vorangegangener UNI-Medientage immer wieder umfassend diskutiert - als auch die Verallgemeinerung der ,heutigen Jugend“ wirft Fragen auf.

Ich erinnere mich an meine eigene Jugend, die zugegebenermaßen schon einige Jahrzehnte her ist. Es hat mich damals sehr gestört, wenn man von der Jugend im Allgemeinen sprach. Denn die eine Jugend gab es damals nicht, und es gibt sie heute noch viel weniger. Gerade in unserer nun deutlich differenzierteren Gesellschaft wird diese Verallgemeinerung virulent. Weltanschaulich, in der Gestaltung der Freizeit, in der Erwartung an ein erfülltes Leben lässt sich immer weniger ein generelles Bild der Jugend zeichnen.

Große Unterschiede zwischen allgemeinen Stehsätzen über die Jugend (Stichwort die Jugend liest keine Zeitung mehr) und der Wirklichkeit sind im Übrigen auch bei der großen Jugend-Studie der Tiroler Tagezeitung im letzten Jahr deutlich geworden. Der oft gehörte Stehsatz: „Die Jugend liest keine Zeitung mehr“ relativiert sich schon allein, wenn man sich die Leserzahlen der Tiroler Tageszeitung (Verein Arbeitsgemeinschaft Medien Analyse 2019) bei den 14- bis 19-Jährigen vergegenwärtigt. Demzufolge lesen rund $30 \%$ der Jugendlichen die Tiroler Tageszeitung. Natürlich gilt auch für diese Studie, dass das Mediennutzungsverhalten von jungen Tirolerinnen und Tirolern nicht beliebig auf das Leserverhalten in anderen Regionen Österreichs oder der Welt schließen lässt.

Katharina Gallner-Holzmann, Theo Hug, Günther Pallaver (Hg.):

Jugendliche Mediennutzung und die Zukunft des Qualitätsjournalismus

(C) 2020 innsbruck university press, ISBN 978-3-99106-014-7, DOI 10.15203/99106-014-7 
Ein Faktum hingegen ist die digitale Transformation, welche die Gesellschaft weltweit erfasst, mit steigender Taktfrequenz. In Österreich beträgt die Verfügbarkeit digitaler Geräte bei Jugendlichen beinahe $100 \%$, ein Großteil der Jugendlichen bezieht auch Nachrichten und Informationen aus Social Media Kanälen. Wenn es jedoch um das Vertrauen in Nachrichten geht, differenziert die Jugend klar: Klassischen Medien wie Tageszeitungen (in Print und online), Radio und Fernsehen wird mehr denn je Vertrauen geschenkt. Bei der Glaubwürdigkeit rangieren Social Media-Kanäle mit Abstand an letzter Stelle.

Gerade die Covid 19-Krise hat die Sehnsucht der Menschen nach vertrauensvollen Informationsquellen verstärkt, über alle Altersgruppen hinweg. Das ist eine erfreuliche Entwicklung, welche die Bedeutung von Qualitätsmedien als essentiellen Bestandteil einer aufgeklärten Gesellschaft vor Augen führt.

Das interfakultäre Medienforum der Universität Innsbruck hat also auch heuer wieder ein spannendes Thema mit Raum für verschiedenste Betrachtungsweisen auf die Agenda gesetzt. Ich bedanke mich bei den Verantwortlichen, insbesondere dem Leiter Theo Hug

und seinem Team für die bewährte Organisation und wünsche viele neue Einsichten und Erkenntnisse.

\section{Literatur}

Verein Arbeitsgemeinschaft Media-Analysen. (2019) MA 2019. Presse. Abgerufen unter: https://www.media-analyse.at/table/3349 [Stand vom 22-06-2020]. 